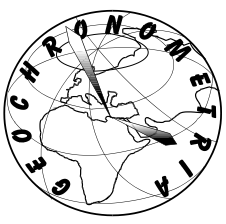

Conference Proceedings of the $5^{\text {th }}$ Asia Pacific Luminescence and Electron Spin Resonance Dating Conference October $15^{\text {th }}-17^{\text {th }}$, 2018, Beijing, China

Guest Editor: Grzegorz Adamiec

\title{
CHARACTERISTICS OF PULSED BLUE AND GREEN LIGHT STIMULATED LUMINESCENCE SIGNALS OF QUARTZ AND FELDSPARS
}

\author{
JINTANG QIN ${ }^{1,3}$, JIE CHEN $^{1,3}$, KECHANG LI ${ }^{1,2,3}$ \\ ${ }^{1}$ State Key Laboratory of Earthquake Dynamics, Institute of Geology, China Earthquake Administration, Beijing 100029, China \\ ${ }^{2}$ Luminescence Dating Laboratory, Institute of disaster prevention, Langfang, Hebei 065201, China \\ ${ }^{3}$ Xinjiang Pamir Intracontinental Subduction National Field Observation and Research Station, Beijing, China
}

Received 13 February 2019

Accepted 30 July 2020

\begin{abstract}
The post-infrared (post-IR) pulsed blue light stimulated luminescence (PBLSL) signal has been employed to determine the equivalent dose $\left(\mathrm{D}_{e}\right)$ of feldspar contaminated quartz grains, but it sometimes suffers from the interference of feldspars. Since the green light stimulated luminescence (GLSL) signal of feldspars might be more reduced by a prior IR stimulation, we compared the characteristics of post-IR PBLSL and post-IR pulsed GLSL (post-IR PGLSL) signals of quartz and feldspars in this study to evaluate the feasibility of employing the green light for pulsed stimulation. We investigated the effect of the signal integration period, pulsed stimulation temperature, and prior IR stimulation temperature on the intensities of post-IR PBLSL and post-IR PGLSL of quartz and feldspars, and evaluated the potential feldspar interference on these two signals for the hypothetical and artificial quartz-feldspar mixture. The results demonstrate a lower feldspars contribution for the post-IR PGLSL signal. The feldspar interference only slightly increases with the increase of integration period for the post-IR PGLSL signal measured at low stimulation temperature, which permits a long integration period to be employed to enhance the signal to noise ratio. This study shows that the green light is a promising alternative for pulsed stimulation to suppress the feldspar contribution.
\end{abstract}

Keywords: pulsed stimulation, green light, low temperature, long integration period.

\section{INTRODUCTION}

The post-IR pulsed blue light stimulated luminescence signal (post-IR PBLSL, Bailiff and Mikhailik, 2003; Denby et al., 2006), which involves pulsing the optical stimulation, was proposed to isolate the luminescence signal dominated by quartz grains in their mixture

Corresponding author: J. Qin

e-mail: jtqin@ies.ac.cn with feldspars. The post-IR PBLSL procedure for determining equivalent dose $\left(\mathrm{D}_{e}\right)$ has been continuously optimised (Thomsen et al., 2008; Ankjærgaard et al., 2010) and applied to samples of a variety of origins (Tsukamoto and Rades, 2016). However, the post-IR PBLSL signal of a quartz-feldspar mixture may still suffer from the contribution from the feldspars and often suffers from the low signal to noise ratio (Ankjærgaard et al., 2009, 2010; Tsukamoto and Rades, 2016).

As an alternative to the blue light stimulation, the green light has long been employed to stimulate the quartz luminescence signals (Bailey et al., 2011), espe- 
cially for early studies on pulsed stimulation (Chithambo and Galloway, 2000; Chithambo, 2003). The response of feldspars to green light stimulation is different from that to blue light stimulation. The trapped electrons are excited into the conduction band and sub-conduction band by the blue light and green light, respectively (Jain and Ankjærgaard, 2011). The recombination of the subconduction band electrons with the holes is significantly modulated by the stimulation temperature, while the recombination of the conduction band electrons is rather less affected by temperature (Poolton et al., 2009). Similarly with the green light stimulation, the IR stimulation also excites the trapped electrons into the sub-conduction band (Jain and Ankjærgaard, 2011). Therefore, it is expected that the electron-hole pairs of feldspars would potentially recombine under the green light stimulation, could be consumed by a prior IR stimulation at elevated temperature (Jain and Singhvi, 2001), and the contribution from feldspars to the post-IR pulsed green light stimulated luminescence (post-IR PGLSL) will be reduced. Such a reduction in the feldspar contribution for the post-IR PGLSL signal may also allow employing long integration periods to improve the signal to noise ratio.

In this study, we tested the idea of using the green light for pulsed stimulation to suppress the feldspar contribution by investigating the characteristics of the postIR PBLSL and post-IR PGLSL signals of both quartz and feldspars. Firstly, we investigated the effect of the signal integration period, pulsed stimulation temperature and prior IR stimulation temperature on the post-IR PBLSL and post-IR PGLSL intensities of quartz and feldspars, and the potential feldspar interference on these two signals for the hypothetical quartz-feldspar mixture. Then, we compared the $\mathrm{D}_{e}$ values determined by applying these two signals to the artificial quartz-feldspar mixture.

\section{SAMPLES, INSTRUMENTS AND METHODS}

\section{Samples}

Samples from a wide geographical coverage are employed in this study (Table 1). Quartz grains with diverse luminescence sensitivities were extracted from four samples, including dune sands from the Qaidam basin
(Q-QB, dim quartz) in western China and the Otindag desert (Q-HD, bright quartz) in eastern China, fluvial sands from the northern Tian Shan piedmont (Q-NTS, dim quartz), and the calibration quartz from the DTU Risø laboratory (Q-CAL, super-bright quartz). Feldspars were extracted from the same fluvial sands mentioned above from the northern Tian Shan piedmont (F-NTS) and the crushed perthite from Shantou of southern China (F-PST). These samples are used to investigate the characteristics of the post-IR PBLSL and post-IR PGLSL signals. Two extra samples from the dune in the Qaidam basin (17YDE2C) and Junggar basin (15MNS01, modern sample) are employed for evaluating the performance of post-IR PBLSL and post-IR PGLSL signals on $\mathrm{D}_{e}$ determination for a quartz-feldspars mixture.

The $125-180 \mu \mathrm{m}$ quartz grains were extracted from the samples Q-QB, Q-HD, Q-NTS and 17YDE2C following the conventional chemical treatments (Aitken, 1998), while the 125-180 $\mu \mathrm{m}$ potassium feldspar (K-feldspar) grains were extracted from the samples F-NTS and $15 \mathrm{MNS} 01$, following the conventional chemical and density separation $(\rho<2.58 \mathrm{~g} / \mathrm{cm} 3)$ procedures, e.g., Buylaert et al. (2009). The perthite (F-PST) was crushed and sieved to extract the $90-125 \mu \mathrm{m}$ fraction, but without further chemical treatment.

\section{Instruments}

The DTU Risø TL/OSL DASH M 20 C/D reader, which is equipped with a $2.96 \mathrm{GBq} \beta$ source, a built-in pulsing unit and photon timer TimeHarp 260, is employed for all luminescence measurements. The full power of the green $(\lambda=525 \mathrm{~nm})$, blue $(\lambda=470 \mathrm{~nm})$, and IR LEDs $(\lambda=870 \mathrm{~nm})$ are $42 \mathrm{~mW} / \mathrm{cm}^{2}, 86 \mathrm{~mW} / \mathrm{cm}^{2}$ and $162 \mathrm{~mW} / \mathrm{cm}^{2}$, respectively. The luminescence signals are stimulated at $90 \%$ of full power and detected by a photomultiplier tube (PMT, module EMD-9107) for all three types of stimulation. For IR stimulation, the blue filter pack (BG3 and BG39) is employed. For the pulsed green light stimulation, only the $5 \mathrm{~mm} \mathrm{U}-340$ filter is used, while both the $5 \mathrm{~mm}$ and $2.5 \mathrm{~mm} \mathrm{U}-340$ filters are employed for the pulsed blue light stimulation. The steel stainless disc is used for mounting on the quartz and feldspar grains.

Table 1. List of samples investigated in this study.

\begin{tabular}{|c|c|c|}
\hline Origin & Mineral & $\begin{array}{c}\text { Grain size } \\
(\mu \mathrm{m})\end{array}$ \\
\hline Qaidam Basin, dune sands & Quartz, Q-QB & $125-180$ \\
\hline Otindag Desert, dune sands & Quartz, Q-HD & $125-180$ \\
\hline Northern Tian Shan, fluvial sands & Quartz, Q-NTS; K-feldspar, F-NTS & $125-180$ \\
\hline DTU Ris $\varnothing$ laboratory & Quartz, Q-CAL & $90-180$ \\
\hline Shantou & Perthite, F-PST & $90-125$ \\
\hline Qaidam Basin, dune sands* & Quartz, 17YDE2C & $125-180$ \\
\hline Junggar Basin, dune sands* & K-feldspar, 15MNS01 & $125-180$ \\
\hline
\end{tabular}

* These two samples were artificially mixed to get the quartz-feldspar mixture. 


\section{Methods}

Firstly, we inspected the build-up and decay characteristics of the time-resolved post-IR PBLSL (TR-post-IR PBLSL) and time-resolved post-IR PGLSL (TR-post-IR PGLSL) signals of quartz and feldspars. Subsequently, the decay characteristic and the variation of intensity and counting error with the integration period were compared for the post-IR PBLSL and post-IR PGLSL signals of both quartz and feldspars. Then, the feldspars interference on the post-IR PBLSL and post-IR PGLSL signals of the hypothetically synthesised quartz-feldspar mixture was evaluated under different IR and pulsed blue or green light stimulation temperature. At last, the $\mathrm{D}_{e}$ values determined by using these two signals were compared by employing an artificially mixed sample with known-dose quartz and feldspar grains.

Two large aliquots $(\Phi=8 \mathrm{~mm})$ were prepared for each sample to investigate the post-IR PBLSL and post-IR PGLSL characteristics (Q-QD, Q-HD, Q-CAL, Q-NTS, F-NTS and F-PST), with their natural signals being bleached by the SOL2 solar simulator for 8 hours. Twenty-four large aliquots $(\Phi=8 \mathrm{~mm})$ were prepared for $\mathrm{D}_{e}$ determination for the quartz-feldspar mixture, which were made of the samples 17YDE2C and 15MNS01.

\section{RESULTS AND DISCUSSION}

\section{TR-post-IR PBLSL and TR-post-IR PGLSL signals}

The time-resolved luminescence measurements were performed to investigate the build-up and decay characteristics of the post-IR PBLSL and post-IR PGLSL signals of all quartz and feldspars aliquots following steps 1-8 in Table 2. Since it is expected that the pulsed green light stimulation at a lower temperature would result in lower contribution from the feldspars, the time-resolved measurements were performed at $25^{\circ} \mathrm{C}$. The detailed experimental parameters are as following: $\mathrm{D}_{\mathrm{R}}=100 \mathrm{~Gy}$, $\mathrm{T}_{\mathrm{IR}}=125^{\circ} \mathrm{C}$ and $\mathrm{T}_{\mathrm{BL}}=\mathrm{T}_{\mathrm{GL}}=25^{\circ} \mathrm{C}$. The on and off period of the pulsing are both $500 \mu \mathrm{s}$.

The build-up and decay characteristics are similar for the TR-post-IR PBLSL and TR-post-IR PGLSL curves of all samples, respectively. Typical curves of a quartz ali-

Table 2. Procedures for investigating the characteristics of post-IR PBLSL and post-IR PGLSL signals.

\begin{tabular}{lll}
\hline Step & Treatment & Observation \\
\hline 1 & Give dose, $\mathrm{D}_{\mathrm{R}} \mathrm{Gy}$ & \\
\hline 2 & Preheat at $260^{\circ} \mathrm{C}$ for $10 \mathrm{~s}$ \\
\hline 3 & IR stimulation for $100 \mathrm{~s}$ at $\mathrm{T}_{\mathrm{IR}}$ \\
\hline 4 & Pulsed blue light stimulation for $200 \mathrm{~s}$ at $\mathrm{T}_{\mathrm{BL}}$ & Post-IR PBLSL \\
\hline 5 & Give dose, $\mathrm{D}_{\mathrm{R}} \mathrm{Gy}$ & \\
\hline 6 & Preheat at $260^{\circ} \mathrm{C}$ for $10 \mathrm{~s}$ \\
\hline 7 & IR stimulation for $100 \mathrm{~s}$ at $\mathrm{T}_{\mathrm{IR}}$ \\
\hline 8 & Pulsed green light stimulation for $200 \mathrm{~s}$ at $\mathrm{T}_{\mathrm{GL}}$ Post-IR PGLSL \\
\hline 9 & Return to step 1 \\
\hline
\end{tabular}

quot of Q-HD and a K-feldspar aliquot of F-NTS are shown in Fig. 1. The blue light stimulation results in two times and six times higher luminescence intensity than the green light stimulation for quartz of Q-HD and K-feldspars of F-NTS, respectively. It means that the luminescence intensity decreases more for the K-feldspar than for the quartz when the blue light stimulation is replaced by the green light stimulation, which helps suppress the feldspar contribution. During the off period, a gradual decaying component is observed for the TR-postIR PBLSL signal of the K-feldspar (Fig. 1b), while an instant decay is observed for the TR-post-IR PGLSL signal (Fig. 1d). It coincides with the observation of Ankjærgaard et al. (2009) that the lifetime of the TRpost-IR PBLSL signal is longer than that of the TR-postIR PGLSL signal for feldspars. Therefore, the delay between the switching off the light source and the onset of signal recording is shortened from $2.5 \mu \mathrm{s}$, which is commonly employed for the post-IR pulsed blue light stimulation, to $0.5 \mu$ s for the post-IR pulsed green light in this study to improve the signal to noise ratio of the post-IR PGLSL signal. Fig. 1 also shows that the build-up of post-IR PBLSL and post-IR PGLSL signals both finish in $50 \mu \mathrm{s}$, therefore, the on and off periods are both taken as $50 \mu$ s for the pulsed stimulation.
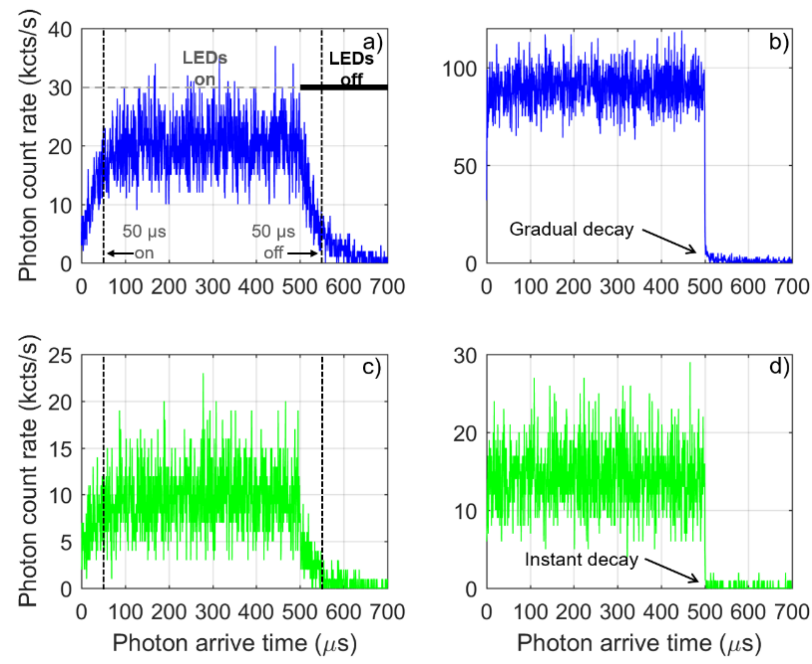

Fig. 1. Time resolved post-IR PBLSL and post-IR PGLSL curves of the quartz and K-feldspars. TR-post-IR PBLSL curves for a) bright quartz of Q-HD and b) K-feldspar of F-NTS, and TR-post-IR PGLSL curves for c) bright quartz of Q-HD and d) K-feldspar of F-NTS. The channel width is $512 \mathrm{~ns}$ and the photon count rate is the integration of signal during the initial $2.4 \mathrm{~s}$ stimulation. The curves are truncated at $700 \mu \mathrm{s}$ for clarity. The vertical dashed lines in a) and c) indicate the timing of the end of on-period and off-period for the subsequent post-IR PBLSL and post-IR PGLSL measurements. 


\section{Post-IR PBLSL and post-IR PGLSL decay characteristics}

Steps $1-8$ of Table 2 were performed to investigate the post-IR PBLSL and post-IR PGLSL decay characteristics for all quartz and feldspar aliquots. For this experiment, the experimental parameters are taken as follows: $\mathrm{D}_{\mathrm{R}}=60 \mathrm{~Gy}, \mathrm{~T}_{\mathrm{IR}}=125^{\circ} \mathrm{C}$ and $\mathrm{T}_{\mathrm{BL}}=\mathrm{T}_{\mathrm{GL}}=25^{\circ} \mathrm{C}$.

The initial intensity of the post-IR PBLSL signal is $\sim$ four to ten times higher than that of the post-IR PGLSL signal for quartz aliquots of super-bright Q-CAL (Fig. 2a), bright Q-HD (Fig. 2b) and $\operatorname{dim}$ Q-QB (Fig. 2c), while the former is $\sim$ seven times higher than the latter for the feldspars of the F-PST (Fig. 2d). The normalised post-IR PBLSL and post-IR PGLSL decay curves show that the post-IR PGLSL signal decays much slower than the post-IR PBLSL signal for quartz aliquots, irrespective of their luminescence sensitivity (Fig. 2e-2g). Fig. 2h shows that the two signals decay at a similar rate for feldspars. It implies that the signal to noise ratio of the post-IR PGLSL signal could be improved by increasing the integration period. Therefore, we investigated the effect of increasing the integration period on the signal to noise ratio.

We adopted different periods to integrate the decay curves shown in Fig. 2a-2c to obtain the signal intensity, with a subtraction of the background intensity integrated within a period of 2.5 times that for the signal. The counting error of the integrated luminescence intensity is calculated following the method of Galbraith (2014), and is used as an index for the signal to noise ratio of the integrated post-IR PBLSL and post-IR PGLSL intensity. The post-IR PGLSL intensity (solid circle) increases significantly with the integration period, while the postIR PBLSL intensity (open circle) shows a rapid increase within the $1.2 \mathrm{~s}$ integration period followed by a slower increase thereafter for the quartz aliquots of Q-CAL and Q-HD (Fig. 3a and 3b). Although a smaller increase is observed for the post-IR PGLSL signal of the dim quartz of Q-QB (Fig. 3c), these data suggest that with the elongated integration period, the post-IR PGLSL intensity is comparable with the post-IR PBLSL intensity. The relative counting error of the integrated post-IR PGLSL intensity (solid square) decreases from $0.4 \%$ to $0.15 \%, 4 \%$ to $1.4 \%$ and $18 \%$ to $5 \%$ with the integration period increasing from $0.4 \mathrm{~s}$ to $6 \mathrm{~s}$ for quartz of super-bright Q-CAL, bright Q-HD and dim Q-QB, respectively. These reduced counting errors are comparable with those of the post-IR PBLSL intensity (open square), for which the counting error only slightly varies with the integration period (Fig. 3). These results demonstrate that the signal to noise ratio of the quartz post-IR PGLSL signal could be substantially improved by increasing the integration period.
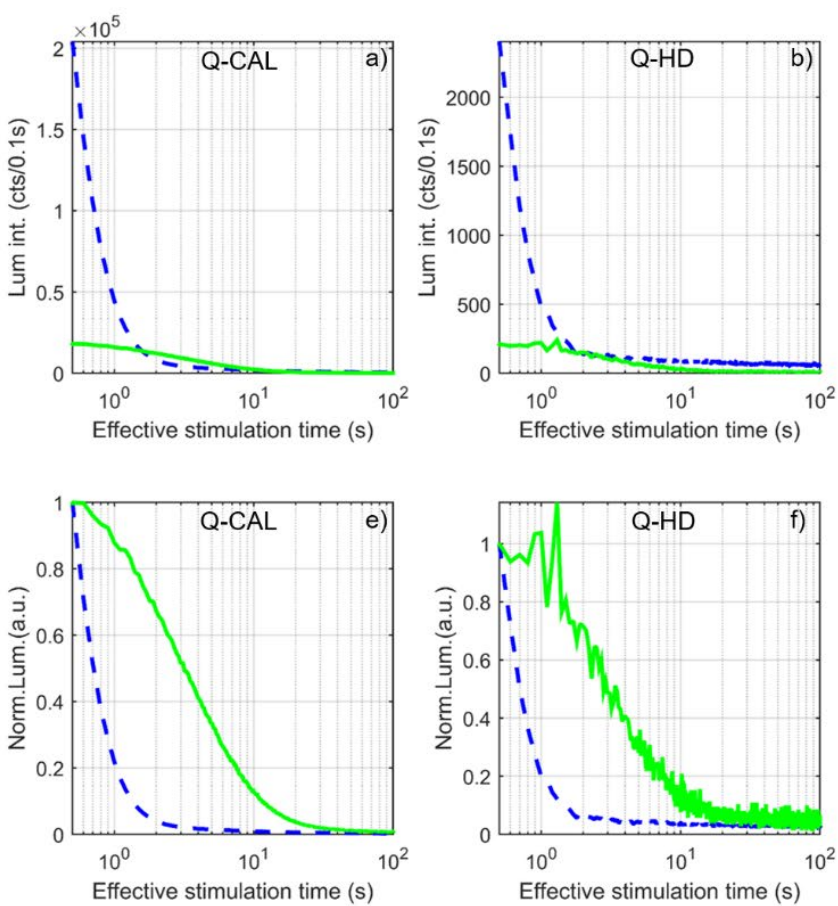
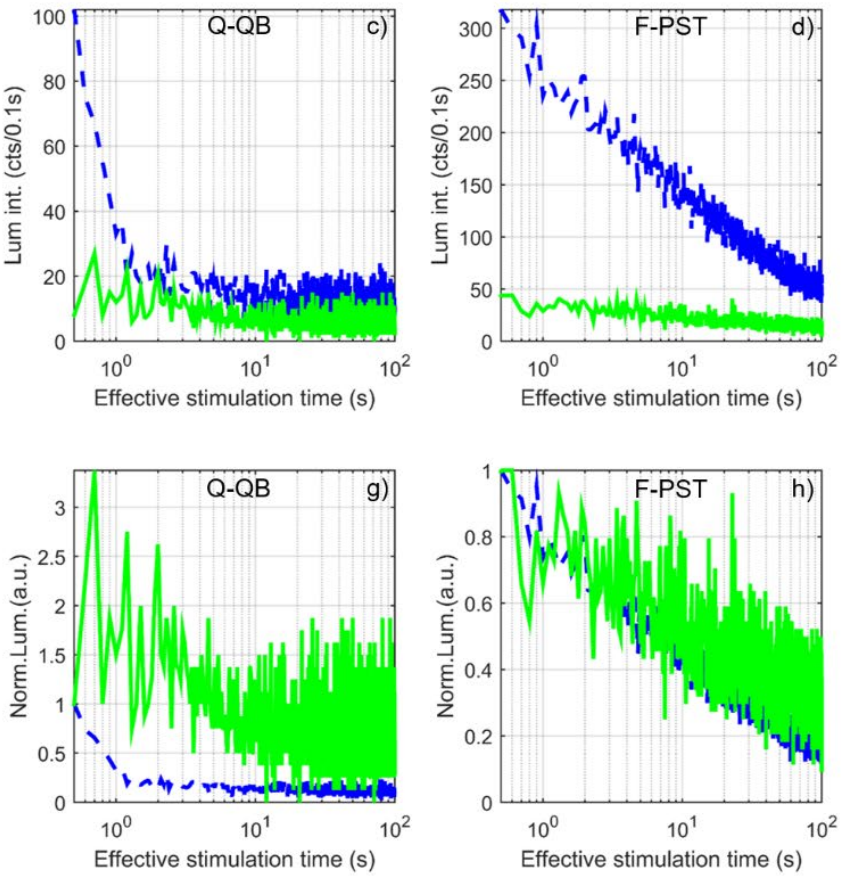

Fig. 2. The raw and normalized post-IR PBLSL (dashed line) and post-IR PGLSL (solid line) decay curves for quartz and feldspars. a) and e) quartz of Q-CAL; b) and f) quartz of Q-HD; c) and g) quartz of Q-QB; d) and h) feldspar of F-PST. The effective stimulation time is calculated by multiplying the ratio of the on period to the sum of on and off period with the total stimulation time. 

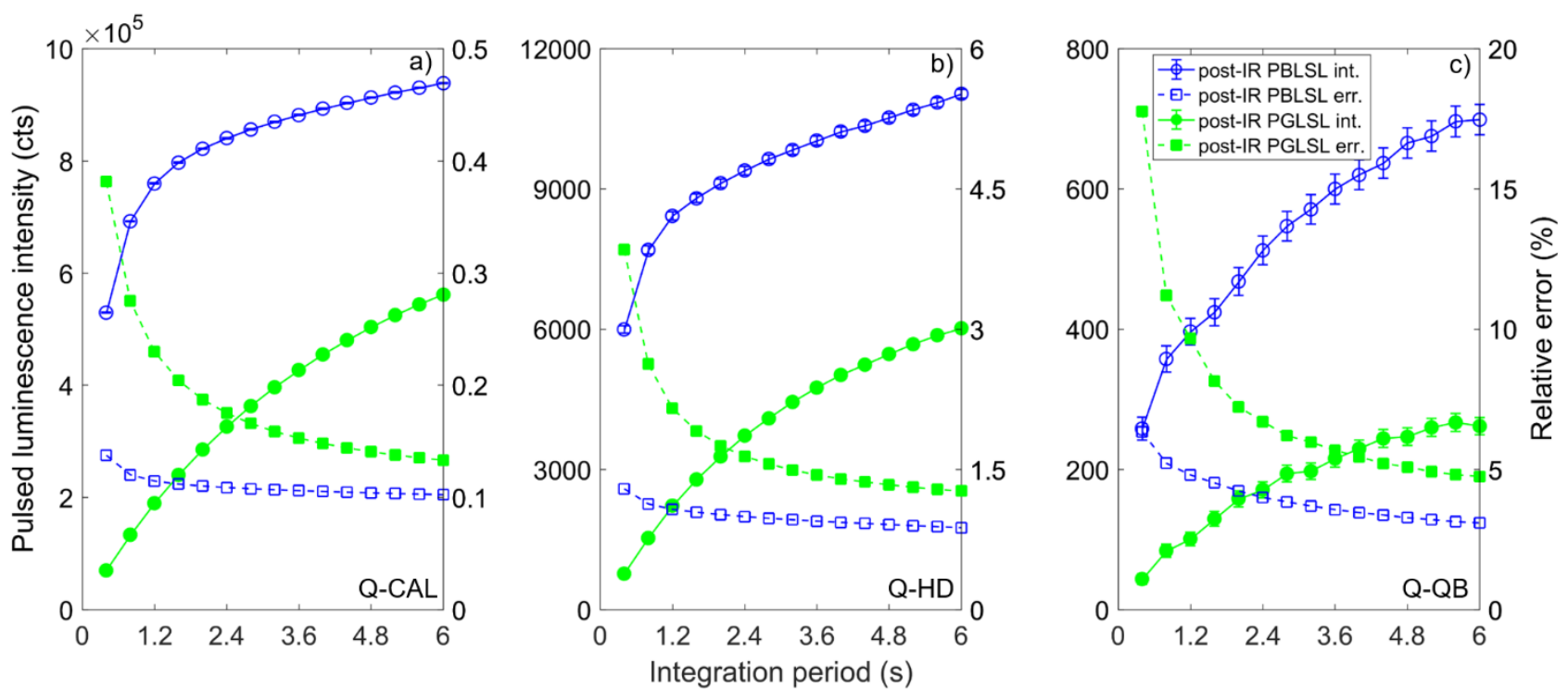

Fig. 3. Variation of the integrated luminescence intensity (circle) and their associated relative counting error (square) for the post-IR PBLSL (open symbol) and post-IR PGLSL (solid symbol) signals of quartz aliquots. a) Q-CAL; b) Q-HD and c) Q-QB.

\section{Variation of post-IR PBLSL and post-IR PGLSL intensity with stimulation temperature}

The effect of stimulation temperature on the luminescence intensity of quartz and feldspars are quite different. With the increase of stimulation temperature, the luminescence intensity generally decreases for the BLSL signal of quartz (Spooner, 1994; Duller et al., 1995; Murray and Wintle, 1998; Bøtter-Jensen et al., 2003), but increases for the PGLSL signal of feldspars (Jain and Ankjærgaard, 2011).

Steps 1-9 of Table 2 were cycled to investigate the effect of stimulation temperature on the post-IR PBLSL and post-IR PGLSL intensity for both quartz and feldspars. The experimental parameters are taken as follows: $\mathrm{D}_{\mathrm{R}}=60 \mathrm{~Gy}, \mathrm{~T}_{\mathrm{IR}}=50^{\circ} \mathrm{C}$, and $\mathrm{T}_{\mathrm{BL}}$ and $\mathrm{T}_{\mathrm{GL}}$ increase from $25^{\circ} \mathrm{C}$ to $200^{\circ} \mathrm{C}$ in an interval of $25^{\circ} \mathrm{C}$. The initial $0.4 \mathrm{~s}$ (effective stimulation time) of the post-IR PBLSL and post-IR PGLSL decay curve is integrated as the signal intensity with a subtraction of the background intensity integrated from the last $1 \mathrm{~s}$ of the decay curve.

The variation of the normalised luminescence intensity with the stimulation temperature is shown in Fig. 4 (each data point is the average of two aliquots measured for each sample). For quartz, the post-IR PBLSL intensity slightly increases with the stimulation temperature increasing from $25^{\circ} \mathrm{C}$ to $75^{\circ} \mathrm{C}$, followed by a decrease up to $80 \%$ with the stimulation temperature further elevated. The post-IR PGLSL intensity increases by about 1.5 to 2 times, with the stimulation temperature increasing up to $125^{\circ} \mathrm{C} \sim 150^{\circ} \mathrm{C}$, followed by a decrease of up to $70 \%$ (Fig. 4a-4d). However, for K-feldspar of F-NTS and perthite of F-PST, the post-IR PGLSL and post-IR PBLSL intensities increase monotonically and more sig-
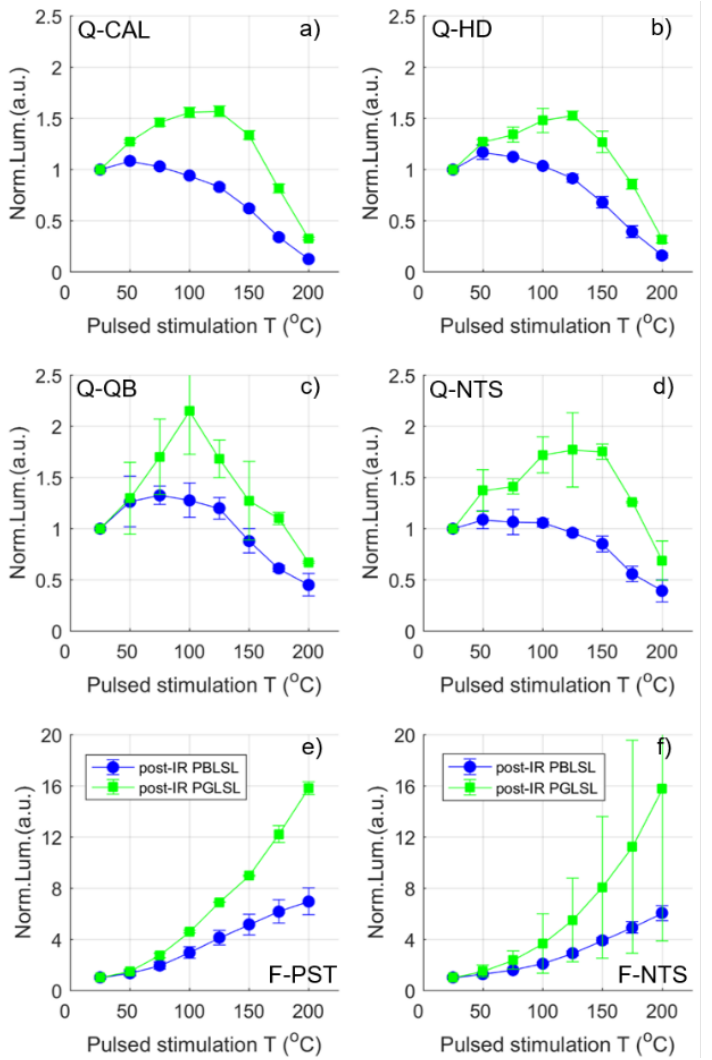

Fig. 4. Variation of the normalized post-IR PBLSL (circle) and post-IR PGLSL (square) intensity on the pulsed stimulation temperature for quartz and feldspars. a) Q-CAL; b) Q-HD; c) Q-QB; d) Q-NTS; e) F-PST and f) F-NTS. The signal intensity was integrated using the initial $0.4 \mathrm{~s}$ effective stimulation time with subtraction of the integration of the last $1 \mathrm{~s}$ effective stimulation time. 
nificantly with the increase of stimulation temperature (Fig. 4e-4f). These results suggest that increasing the stimulation temperature help increase quartz post-IR PGLSL intensity, but at the expense of more contribution from the feldspars if the post-IR PGLSL signal of the quartz-feldspar mixture were measured.

To quantify the relative contribution of the feldspars to the total luminescence signal of the quartz-feldspar mixture, the post-IR PBLSL and post-IR PGLSL decay curves of a quartz aliquot and a feldspar aliquot were summed as the post-IR PBLSL and post-IR PGLSL decay curves of a hypothetical aliquot of the quartz-feldspar mixture, respectively. Subsequently, the luminescence intensities were calculated for the post-IR PBLSL (PGLSL) signal of the feldspars $\left(\mathrm{L}_{\mathrm{f}}\right)$ and the mixture $\left(\mathrm{L}_{\mathrm{q}+\mathrm{f}}\right)$, respectively, by employing different integration periods. The ratio of $\mathrm{L}_{\mathrm{f}}$ to $\mathrm{L}_{\mathrm{q}+\mathrm{f}}\left(\mathrm{L}_{\mathrm{f}} / \mathrm{L}_{\mathrm{q}+\mathrm{f}}\right)$ is taken as the relative contribution of feldspars.

Fig. 5 and Fig. 6 show the dependence of $L_{f} / L_{q}+f$ on the integration period under various stimulation temperature for the hypothetic mixture of the feldspar with bright quartz Q-HD and dim quartz Q-NTS, respectively. For the hypothetic mixture with bright quartz of Q-HD, the feldspar contribution to the post-IR PGLSL signal shows a negligible increase with the integration period at low stimulation temperature, while a sharp increase is observed for the post-IR PBLSL signal (Fig. 5). With elevating the stimulation temperature, the feldspar contribution increases significantly with the integration period for the post-IR PGLSL signal. With the same integration period, the feldspar contribution increases with the stimulation temperature for the post-IR PGLSL signal. Fig. 5 unambiguously shows that the feldspar contribution to the post-IR PGLSL signal is lower than that to the postIR PBLSL signal at any stimulation temperature, especially at low temperature. For the hypothetic mixture with dim quartz of Q-NTS, the feldspar contribution to the post-IR PGLSL signal is also the lowest and shows little dependence on the integration period at $25^{\circ} \mathrm{C}$ (Fig. 6), but the absolute feldspar contribution is much higher than that the mixture with Q-HD. Therefore, the low stimulation temperature, e.g., $25^{\circ} \mathrm{C}$, is preferred for pulsed green light stimulation to suppress the feldspar contribution.
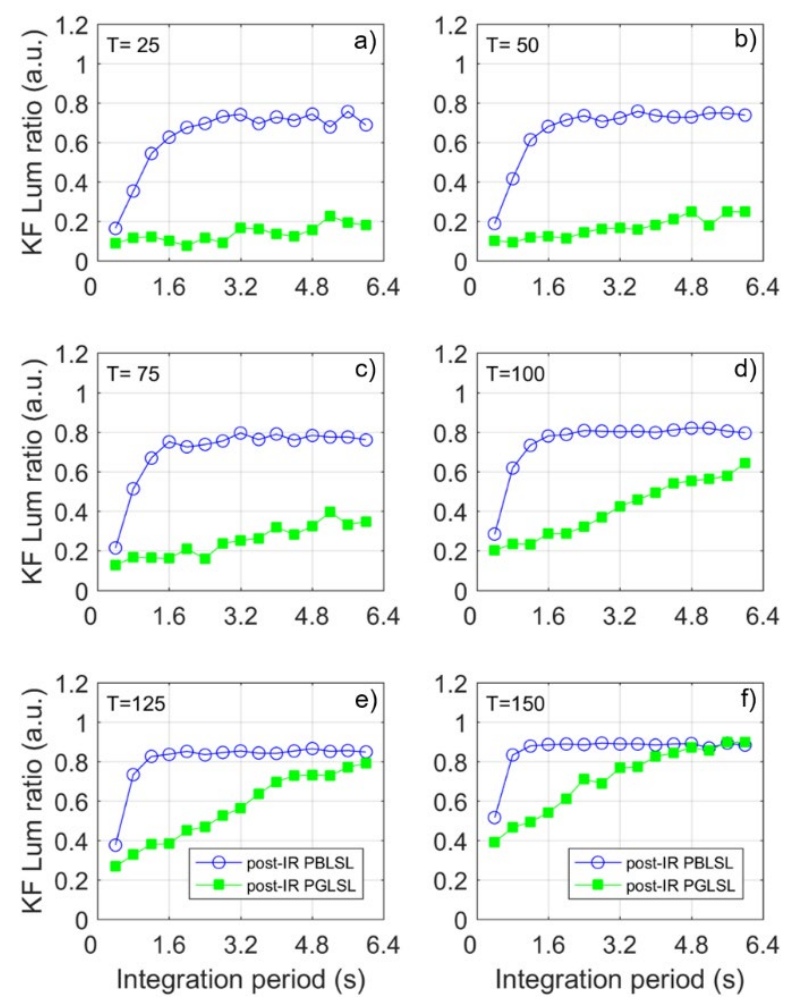

Fig. 5. Variation of the relative feldspar contribution to the post-IR PBLSL (open circle) and post-IR PGLSL (solid square) signals with the integration period for the hypothetical quartz (Q-HD) and feldspar (F-NTS) mixture under various pulsed stimulation temperatures. a) $25^{\circ} \mathrm{C}$; b) $50^{\circ} \mathrm{C}$; c) $75^{\circ} \mathrm{C}$; d) $100^{\circ} \mathrm{C}$; e) $125^{\circ} \mathrm{C}$ and f) $150^{\circ} \mathrm{C}$.
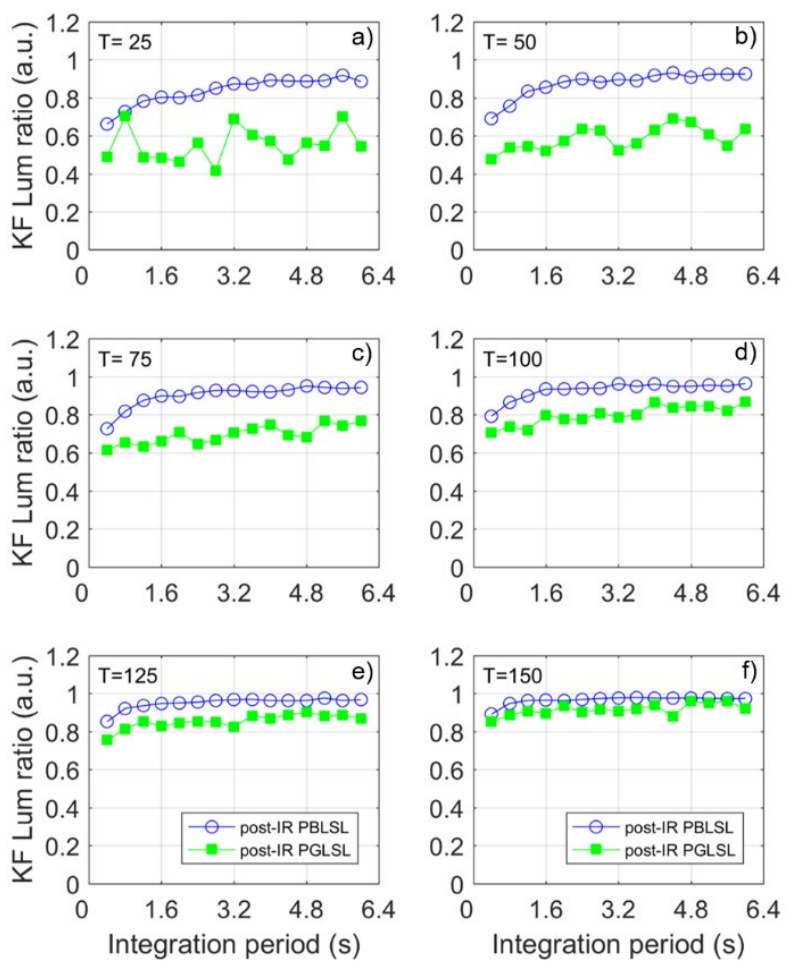

Fig. 6. Variation of the relative feldspar contribution to the post-IR PBLSL (open circle) and post-IR PGLSL (solid square) signals with the integration period for the hypothetical quartz (Q-NTS) and feldspar (F-NTS) mixture under various pulsed stimulation temperatures. a) $25^{\circ} \mathrm{C}$; b) $50^{\circ} \mathrm{C}$; c) $75^{\circ} \mathrm{C}$; d) $100^{\circ} \mathrm{C}$; e) $125^{\circ} \mathrm{C}$ and f) $150^{\circ} \mathrm{C}$. 


\section{Effect of IR stimulation temperature on feldspar con- tribution}

Increasing the IR stimulation temperature has dual effects on the feldspars contribution to the post-IR PGLSL signals. On the one hand, it would help remove more trapped electrons sensitive to the green light stimulation for the feldspars, since more electron-hole pairs contributing to post-IR PGLSL signal would be consumed by the prior IR stimulation; one the other hand, the hightemperature IR stimulation, e.g., higher than $120^{\circ} \mathrm{C}$, may deplete the fast component BLSL signal of quartz (Jain et al., 2003). Therefore, the effect of IR stimulation temperature on the contribution of feldspar to the post-IR PGLSL signal needs to be investigated.

Steps 1-9 of Table 2 were applied to all quartz and feldspar aliquots for this experiment, with $\mathrm{D}_{\mathrm{R}}=60 \mathrm{~Gy}, \mathrm{~T}_{\mathrm{BL}}$ $=\mathrm{T}_{\mathrm{GL}}=25^{\circ} \mathrm{C}$. These steps were repeated with the IR stimulation temperature $\mathrm{T}_{\mathrm{IR}}$ increasing from $50^{\circ} \mathrm{C}$ to $225^{\circ} \mathrm{C}$ in an interval of $25^{\circ} \mathrm{C}$. The initial $0.4 \mathrm{~s}$ (effective stimulation time) of the post-IR PBLSL and post-IR PGLSL decay curves were integrated as the signal intensity with a subtraction of the last $1 \mathrm{~s}$ (effective stimulation time) integration as background. The averaged intensity of two aliquots of one sample was taken for analysis.

The post-IR PBLSL and post-IR PGLSL intensities of quartz do not change much with the prior IR stimulation temperature increasing from $50^{\circ} \mathrm{C}$ to $125^{\circ} \mathrm{C}$, but decreases by $50 \%$ with the temperature further increased to $225^{\circ} \mathrm{C}$ (Fig. 7a-7d). For the feldspars, the post-IR PBLSL and post-IR PGLSL intensity monotonically decrease by up to $60 \%$ with the prior IR stimulation temperature increasing from $50^{\circ} \mathrm{C}$ to $125^{\circ} \mathrm{C}$, followed by a further decrease by $30 \%$ (Fig. 7e and 7f). To balance the reduction of post-IR PGLSL intensity for both quartz and feldspars, the IR stimulation temperature of $125^{\circ} \mathrm{C}$ is preferred for the dim quartz, while it might be increased for the bright quartz as long as the signal to noise ratio is acceptable.

\section{Equivalent dose measurements}

The above results demonstrate that the post-IR pulsed green light stimulation performs better on isolating quartz dominated luminescence signal than the post-IR pulsed blue light stimulation. In this session, their performances on suppressing the feldspar interferences were evaluated by employing both two signals to determine the $\mathrm{D}_{e}$ value of the artificial quartz-feldspar mixture.

For this experiment, the quartz grains extracted from the dune sands of 17YDE2C were mixed with the $\mathrm{K}$-feldspar grains extracted from the modern dune sands of $15 \mathrm{MNS} 01$, in a mass ratio of $2: 1$. The $\mathrm{D}_{e}$ value obtained by using the conventional BLSL signal is $\sim 120$ Gy for the quartz of $17 \mathrm{YDE} 2 \mathrm{C}$, while the $\mathrm{D}_{e}$ value of the modern K-feldspar grains is $\sim 0$ Gy. The single aliquot regenerative dose (SAR) based pulsed stimulation procedure (Tsukamoto and Rades, 2016) was employed to determine the $\mathrm{D}_{e}$ value of the mixture. The preheat and cut-heat are $260^{\circ} \mathrm{C}-10 \mathrm{~s}$ and $220^{\circ} \mathrm{C}-5 \mathrm{~s}$, respectively. The pulsed (50 $\mu$ s on period and $50 \mu$ s off period) blue or green light stimulation for $200 \mathrm{~s}$, at either $25^{\circ} \mathrm{C}$ (suggested by this study) or $125^{\circ} \mathrm{C}$ (conventionally employed), was performed following a prior IR stimulation at $125^{\circ} \mathrm{C}$ for $100 \mathrm{~s}$. Twelve aliquots were measured for each procedure.

The median of post-IR PGLSL $\mathrm{D}_{e}$ measured at $25^{\circ} \mathrm{C}$ is $\sim 80$ Gy and almost independent of the integration period (Fig. 8a), while the median of post-IR PBLSL $\mathrm{D}_{e}$ decreases significantly from $\sim 40$ Gy to $\sim 10$ Gy with the increase of integration period (Fig. 8b). With the stimulation temperature increased to $125^{\circ} \mathrm{C}$, the median of postIR PGLSL $\mathrm{D}_{e}$ is $\sim 80$ Gy with the effective integration period of $0.4 \mathrm{~s}$ but decreases gradually to $40 \mathrm{~Gy}$ with the effective integration period increased to $6 \mathrm{~s}$ (Fig. 8c). The post-IR PBLSL $\mathrm{D}_{e}$ values measured at $125^{\circ} \mathrm{C}$ are much lower than those measured at $25^{\circ} \mathrm{C}$, and tend to be zero with the elongated integration period (Fig. 8d). The ratio of the post-IR PGLSL $\mathrm{D}_{e}$ values to the post-IR PBLSL D values are almost two with the short integration period,
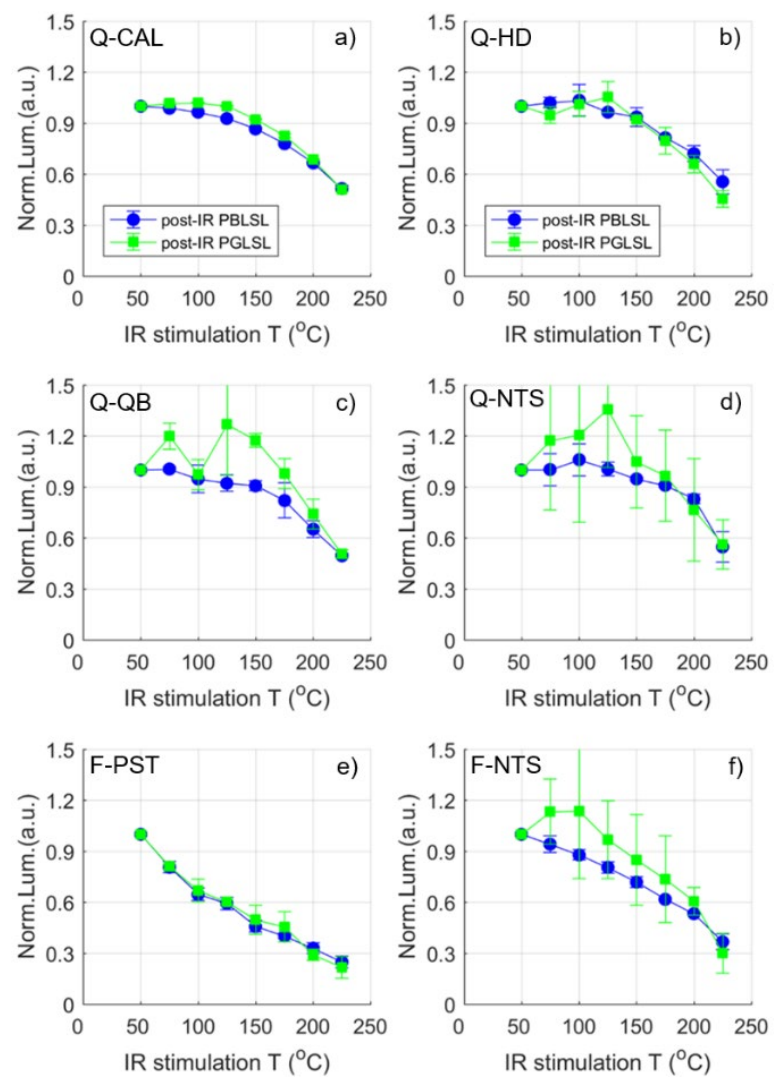

Fig. 7. Variation of the normalized post-IR PBLSL (circle) and post-IR $P G L S L$ (square) intensity with the temperature of IR stimulation prior to the pulsed stimulation for quartz and feldspars. a) Q-CAL; b) Q-HD; c) Q-QB; d) Q-NTS; e) F-PST and f) F-NTS. The signal intensity was integrated using the initial $0.4 \mathrm{~s}$ effective stimulation time with subtraction of the integration of the last $1 \mathrm{~s}$ effective stimulation time. 

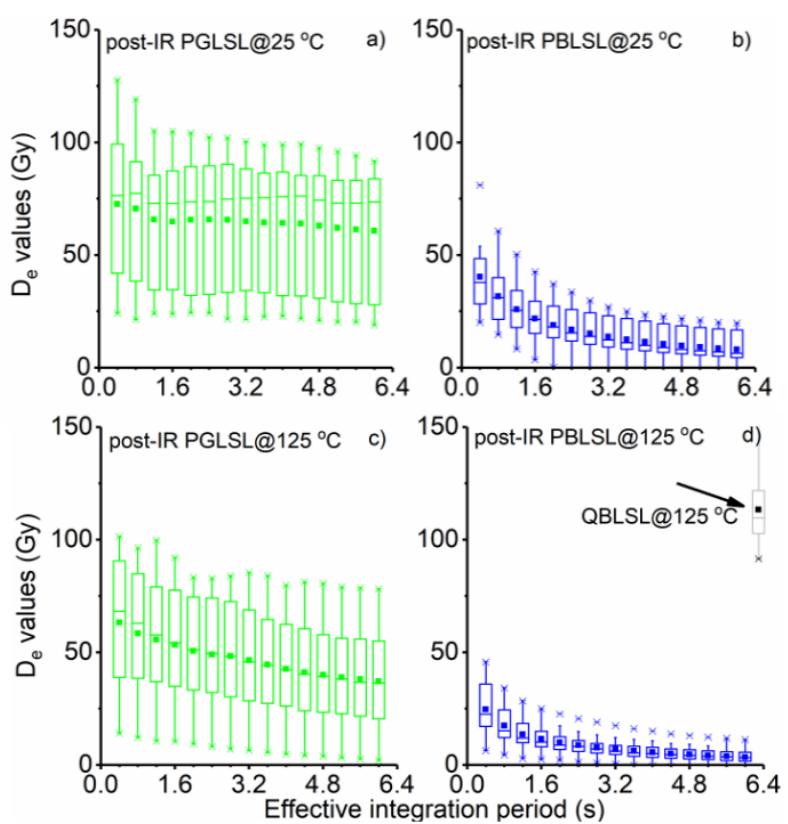

Fig. 8. Box plots of $D_{e}$ values measured by various measurement procedures. a) post-IR PGLSL and b) post-IR PBLSL stimulated at $25^{\circ} \mathrm{C}$; c) post-IR PGLSL and d) post-IR PBLSL stimulated at $125^{\circ} \mathrm{C}$. The $D_{\mathrm{e}}$ value determined by the conventional BLSL signal of quartz of $17 Y D E 2 C$ is shown in d).

while such ratio further increases with the more elongated integration period. These results show unambiguously that the post-IR PGLSL signal performs much better than the post-IR PBLSL signal on determining $\mathrm{D}_{e}$ values of feldspars contaminated quartz grains.

However, the post-IR PGLSL $\mathrm{D}_{e}$ is still one third lower than the $\mathrm{D}_{e}$ value determined by pure quartz grains. The feldspar contribution is much more exaggerated for this artificially mixed sample than for the natural sample, with respect to the feldspar mass ratio and the $\mathrm{D}_{e}$ difference between quartz and feldspar of the natural samples. The impact of the medium and slow component on $\mathrm{D}_{e}$ determination needs to be investigated in future if the elongated integration period is employed. The effect of shallow trap re-trapping on $\mathrm{D}_{e}$ determination at low stimulation temperature also needs to be considered with respect to the signal stability, dose recovery ratio as well as the $\mathrm{D}_{e}$ dispersion (Murray and Wintle, 2000). If the stimulation temperature has to be as high as $125^{\circ} \mathrm{C}$, the prior IR stimulation temperature might be further elevated correspondingly to eliminate the electron-hole pairs contributing to the post-IR PGLSL signal. It is also worthwhile to investigate the feasibility of increasing the power of the green light source of the luminescence reader or even using the post-IR continuous green light stimulation to measure $\mathrm{D}_{e}$ values of feldspar contaminated quartz sample.

\section{CONCLUSION}

We investigated the post-IR PBLSL and post-IR PGLSL characteristics of quartz and feldspar grains of a variety of origins in this study. Our results demonstrate that the feldspar contribution is, in general, lower for the post-IR PGLSL signal than for the post-IR PBLSL signal of the quartz-feldspar mixture. At low stimulation temperature, e.g., $25^{\circ} \mathrm{C}$, the relative feldspars contribution is independent of the integration period for the post-IR PGLSL signal, which enables a long integration period to be adopted to improve the signal to noise ratio. The $\mathrm{D}_{e}$ values measured for the artificial quartz-feldspar mixture by using the post-IR PGLSL signal are almost twice of those determined using the post-IR PBLSL signal and closer to the expected $\mathrm{D}_{e}$ values, under both low $\left(25^{\circ} \mathrm{C}\right)$ and high $\left(125^{\circ} \mathrm{C}\right)$ stimulation temperature. Therefore, we propose to employ the post-IR PGLSL signal for $\mathrm{D}_{e}$ determination for the feldspar contaminated quartz samples, although further investigation on the effect of re-trapping at low stimulation temperature is still needed.

\section{ACKNOWLEDGEMENT}

This study is supported by the grants of the State Key Laboratory of Earthquake Dynamics (LED2016A07), the National Natural Science Foundation of China (41671008), and the Institute of Geology, China Earthquake Administration (IGCEA1810). We thank $\mathrm{Mr}$ Fengyue Qiu for providing the perthite and Professor Lupeng $\mathrm{Yu}$ for providing the dune sample 17YDE2C. We appreciate the detailed comments from an anonymous reviewer, which help improve the manuscript.

\section{REFERENCES}

Aitken MJ, 1998. An Introduction to Optical Dating. Oxford University Press, Oxford.

Ankjærgaard C, Jain M, Kalchgruber R, Lapp T, Klein D, McKeever SWS, Murray AS and Morthekai P, 2009. Further investigations into pulsed optically stimulated luminescence from feldspars using blue and green light. Radiation Measurements 44: 576-581, DOI 10.1016/j.radmeas.2009.02.017.

Ankjærgaard C, Jain M, Thomsen KJ and Murray AS, 2010. Optimising the separation of quartz and feldspar optically stimulated luminescence using pulsed excitation. Radiation Measurements 45: 778775, DOI 10.1016/j.radmeas.2010.03.004.

Bailey RM, Yukihara EG and McKeever SWS, 2011. Separation of quartz optically stimulated luminescence components using green (525 nm) stimulation. Radiation Measurements 46: 643-648, DOI 10.1016/j.radmeas.2011.06.005.

Bailiff IK and Mikhailik VB, 2003. Spatially-resolved measurement of optically stimulated luminescence and time-resolved luminescence. Radiation Measurements 37: 151-159, DOI 10.1016/S1350-4487(02)00187-7.

Bøtter-Jensen L, McKeever SWS and Wintle AG, 2003. Optically Stimulated Luminescence Dosimetry. Elsevier, Amsterdam, pp.355.

Buylaert JP, Murray AS, Thomsen KJ and Jain M, 2009. Testing the potential of an elevated temperature IRSL signal from K-feldspar. 
Radiation Measurements 44: 560-565, DOI 10.1016/j.radmeas.2009.02.007.

Chithambo ML, 2003. Dependence of the thermal influence on luminescence lifetimes from quartz on the duration of optical stimulations. Radiation Measurements 37: 167-175, DOI 10.1016/S13504487(02)00166-X.

Chithambo ML and Galloway RB, 2000. On luminescence lifetimes in quartz. Radiation Measurements 32: 621-626, DOI 10.1016/S1350-4487(00)00096-2.

Denby PM, Bøtter-Jensen L, Murray AS, Thomsen KJ and Moska P, 2006. Application of pulsed OSL to the separation of the luminescence components from a mixed quartz/feldspar sample. Radiation Measurements 41: 774-779, DOI 10.1016/j.radmeas.2006.05.017.

Duller GAT, Bøtter-Jensen L and Poolton NRJ, 1995. Stimulation of mineral-specific luminescence from multi-mineral samples. Radiation Measurements 24: 87-93, DOI 10.1016/1350-4487(94)00098-L.

Galbraith R, 2014. A further note on the variance of a backgroundcorrected OSL count. Ancient TL 32(1): 1-3.

Jain M and Ankjaergaard C, 2011. Towards a non-fading signal in feldspar: Insight into charge transport and tunnelling from timeresolved optically stimulated luminescence. Radiation Measurements 46: 292-309, DOI 10.1016/j.radmeas.2010.12.004.

Jain $\mathrm{M}$ and Singhvi AK, 2001. Limits to depletion of blue-green light stimulated luminescence in feldspars: implications for quartz dating. Radiation Measurements 33: 883-892, DOI 10.1016/S13504487(01)00104-4.
Jain M, Murray AS and Bøtter-Jensen L, 2003. Characterisation of bluelight stimulated luminescence components in different quartz samples: implications for dose measurement. Radiation Measurements 37: 441-449, DOI 10.1016/S1350-4487(03)00052-0.

Murray AS and Wintle AG, 1998. Factors controlling the shape of the OSL decay curve in quartz. Radiation Measurements 29: 65-79, DOI 10.1016/S1350-4487(97)00207-2.

Murray AS and Wintle AG, 2000. Luminescence dating of quartz using an improved single-aliquot regenerative-dose protocol. Radiation Measurements 32: 57-73, DOI 10.1016/S1350-4487(99)00253-X.

Poolton NRJ, Kars RH, Wallinga J and Bos AAJ, 2009. Direct evidence for the participation of band-tails and excited-state tunnelling in the luminescence of irradiated feldspars. Journal of Physics: Condensed Matter 21(48): 485505, DOI 10.1088/0953$8984 / 21 / 48 / 485505$.

Spooner NA, 1994. On the optical dating signal from quartz. Radiation Measurements 23: 593-600, DOI 10.1016/1350-4487(94)90105-8.

Thomsen KJ, Jain M, Murray AS, Denby PM, Roy N and Bøtter-Jensen L, 2008. Minimizing feldspar OSL contamination in quartz UVOSL using pulsed blue stimulation. Radiation Measurements 43: 752-757, DOI 10.1016/j.radmeas.2008.01.020.

Tsukamoto S and Rades EF, 2016. Performance of pulsed OSL stimulation for minimising the feldspar signal contamination in quartz samples. Radiation Measurements 84: 26-33, DOI 10.1016/j.radmeas.2015.11.007. 